\title{
Reduced-Order Predictive Outage Compensators for Networked Systems
}

\author{
Erik Henriksson
}

\author{
Henrik Sandberg
}

Karl Henrik Johansson

\begin{abstract}
Control systems utilizing wireless sensor and actuator networks can be severely affected by the properties of the wireless links. Radio fading and interference may cause communication outage of several samples in situations when the radio environment is noisy and low transmission power is desirable. We propose a method to compensate for outages by introducing a predictive outage compensator (POC), which is a filter to be implemented at the receiver sides of networked control systems and that generates artificial samples during the outage. The main contribution of the paper is to show that a POC can be derived based on a Kalman filter formulation and that it is possible to achieve good performance with a loworder implementation based on Hankel norm approximation. Tradeoffs between achievable closed-loop performance, outage length, and POC order are discussed. The results are illustrated on a simulated example of a multiple-tank process.
\end{abstract}

\section{INTRODUCTION}

Recent advances in low-power wireless radio and sensor technologies have enabled the engineering of a new type of networked sensing and control systems, which are now being tested and evaluated in process industry. In these wireless control systems, it is hard to prevent communication outages to occur due to noisy radio environments. The outages correspond to short time intervals during which sensor data do not reach the controller node or control commands do not reach the actuator node. The approach taken in this paper is to investigate possibilities to compensate for outages using software components in network devices. The work is particularly motivated by a case study within the SOCRADES project [1], where wireless control of the floatation tanks in an ore concentrator at Boliden in Sweden is studied.

Consider the networked control system in Fig. 1, which shows how actuators, sensors and controllers are being connected through a wireless network. Sensors and controllers use a medium access control (MAC) protocol to decide when to transmit sensor and control data over the network. These data are received by the controllers and actuators. At the input to each such device there is a predictive outage compensator (POC), which is a filter that can generate artificial samples during outage. If the MAC and POC are working appropriately, they allow us to abstract away the details of the network in the control design.

The main contribution of the paper is to show that a POC can be derived based on a Kalman filter formulation, which yield an optimal outage prediction under certain assumptions.

This work was supported by the European Integrated Project SOCRADES, the Swedish Research Council, the Swedish Governmental Agency for Innovation Systems, and the Swedish Foundation for Strategic Research.

E. Henriksson, H. Sandberg and K. H. Johansson are with the ACCESS Linnaeus Centre, School of Electrical Engineering, Royal Institute of Technology, 10044 Stockholm, Sweden \{erike02, hsan, kallej\}@ee.kth.se

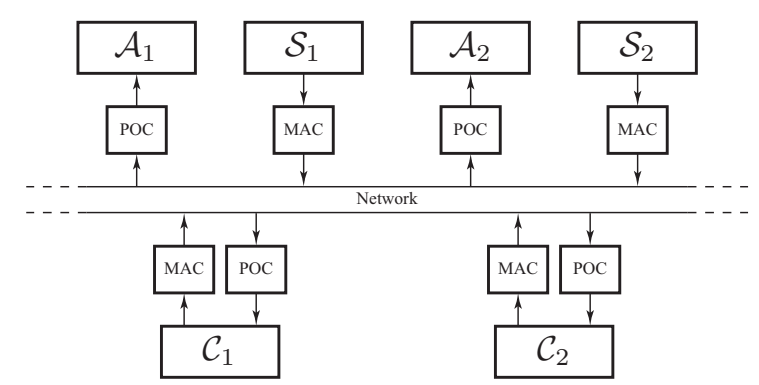

Fig. 1. Actuator, sensor and control devices are interfaced to the network through MAC and POC protocols

In general, such an optimal POC can be of high order. We show that it is possible to achieve good performance with a low-order implementation based on Hankel-norm approximation. A special case of our formulation is the commonly used zero-order hold compensator, which simply holds the last control command during the outage.

Networked control under lossy communication links is a very active research area. Relevant approaches include networking protocols suitable for control [2], [3], compensation schemes for control and estimation algorithms [4], [5], [6], [7], and joint communication and control designs [8], [9]. Issues regarding stabilization of systems using smart actuators for a given drop probability are handled in [10]. Predictive control has been extensively used in various networked control settings, e.g., [11], [12]. The POC was introduced in [13], where we showed that a fairly simple filter significantly could improve the performance of control systems under communication outages.

The outline of the paper is as follows. In Section II the problem is defined. Section III characterizes the optimal POC. Methods to device reduced order POCs and their error bounds are given in Section IV. The method is exemplified and evaluated on a simulated example in Section V. Concluding discussions and future work are given in Section VI.

Notation: We have tried to use standard notation in the paper. Let $\ell_{2}$ denote the Hilbert space of squaresummable signals, i.e., signals $u$ with finite norm $\|u\|_{2}:=$ $\sqrt{\sum_{i=-\infty}^{\infty}|u(i)|^{2}}$. By $\hat{x}\left(k \mid k^{\prime}\right)$ we denote an estimate of $x(k)$, given all available measurements up until time $k^{\prime}$.

\section{Problem Formulation}

In this paper, we consider the problem of controlling a linear plant $\mathcal{P}$ over a communication network with sporadic outages. The plant $\mathcal{P}$ is given by

$$
\begin{aligned}
x_{p}(k+1) & =A_{p} x_{p}(k)+B_{u} u(k)+B_{d} d(k) \\
y(k) & =C_{p} x_{p}(k)+v(k),
\end{aligned}
$$




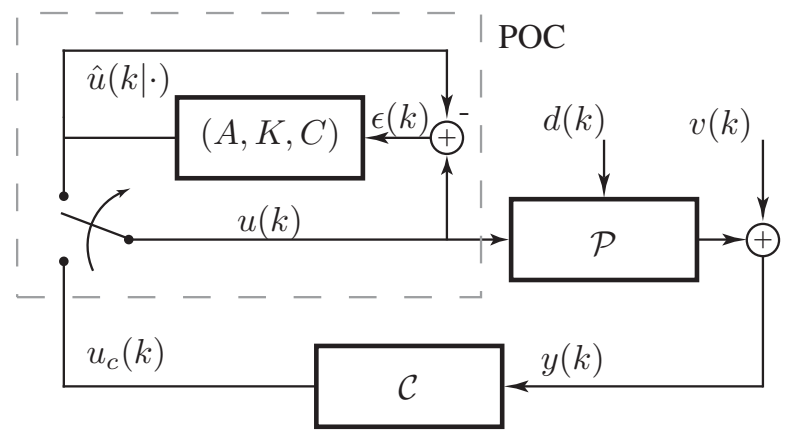

Fig. 2. The Predictive Outage Compensator.

where $d, v$ are process and measurement noise, respectively. When there is no communication outage, the control $u(k)=$ $u_{c}(k)$ is given by the controller $\mathcal{C}$,

$$
\begin{aligned}
x_{c}(k+1) & =A_{c} x_{c}(k)+B_{c} y(k) \\
u_{c}(k) & =C_{c} x_{c}(k) .
\end{aligned}
$$

When there is a communication outage, the actuator does not receive the controller output $u_{c}(k)$. The problem we consider is how one during communication outage should choose $u(k)$. The design of the MAC is left outside the scope of the paper. Further, we assume that the communication between sensor and controller always is working.

The POC is designed to generate controls $u(k)$ when $u_{c}(k)$ is not received. We assume the POC takes the state-space form

$$
\begin{aligned}
& \hat{x}(k+1 \mid k)=A \hat{x}(k \mid k-1)+K \epsilon(k) \\
& \hat{u}(k \mid k-1)=C \hat{x}(k \mid k-1),
\end{aligned}
$$

where $\epsilon(k)=u_{c}(k)-\hat{u}(k \mid k-1)=u_{c}(k)-C \hat{x}(k \mid k-1)$ is the one-step-ahead prediction error of the POC, and $\hat{u}(k \mid k-1)$ is the predicted value of $u_{c}(k)$. The matrices $A, K, C$ are design parameters. A standing assumption in the paper is that $A-K C$ is a Schur matrix so that (3) is an asymptotically stable system.

Equation (3) describes the POC when there is no communication outage, i.e., in "correction mode". When an outage occurs, say at time $k^{\prime}$, the POC switches to "prediction mode" and applies its prediction as input to the plant for $k>k^{\prime}$

$$
\begin{aligned}
\hat{x}\left(k+1 \mid k^{\prime}\right) & =A \hat{x}\left(k \mid k^{\prime}\right) \\
u(k)=\hat{u}\left(k \mid k^{\prime}\right) & =C \hat{x}\left(k \mid k^{\prime}\right)
\end{aligned}
$$

The operation of the POC is illustrated in Fig. 2. In outage, the plant will evolve according to

$$
\begin{aligned}
x_{p, \text { out }}(k+1) & =A_{p} x_{p, \text { out }}(k)+B_{u} \hat{u}\left(k \mid k^{\prime}\right)+B_{d} d(k) \\
y_{\text {out }}(k) & =C_{p} x_{p, \text { out }}(k)+v(k),
\end{aligned}
$$

for $k>k^{\prime}$ when the POC is used. We make the standing assumption that the disturbance $d(k)$ is independent of the outage. The effect of the outage on the plant becomes

$$
\Delta x_{p}(k+1)=A_{p} \Delta x_{p}(k)+B \Delta u(k),
$$

where $\Delta u(k)=u_{c}(k)-\hat{u}\left(k \mid k^{\prime}\right)$ and $\Delta x_{p}(k)=x_{p}(k)-$ $x_{p, \text { out }}(k)$. To minimize the effect of the outage, we would like to make $\Delta u(k)$ as small as possible. How large $\Delta u(k)$ becomes of course depend on how the realization $A, K, C$ is chosen in (4).
In Section III, we characterize POCs that minimize the disturbance $\mathbf{E}|\Delta u(k)|^{2}$ on the plant. We call these optimal POCs. In Section IV, we then show how POCs can be approximated.

Remark 1: We here assume that the POC only gets access to the controller output $u_{c}(k)$ (when available). One could extend the framework and let it have access to more data, such as the controller state. An advantage with the current set-up is that it does not require any modification of the controller $\mathcal{C}$.

Remark 2: If the system (5) is open-loop unstable even a small $\Delta u(k)$ will cause the state $\Delta x_{p}(k)$ to diverge. Hence we can conclude that all open-loop unstable systems are inherently difficult to handle.

\section{Optimal Predictive Outage Compensation}

In this section, we characterize an optimal POC, given that we know the models of $\mathcal{P}$ and $\mathcal{C}$, and know a stochastic model of the noise. Even if these assumptions are not always true, it is still interesting to characterize the optimal solution since it can be used for comparison with other solutions.

Let us first assume that the colored process noise $d(k)$ is given by

$$
\begin{aligned}
x_{d}(k+1) & =A_{d} x_{d}(k)+B_{w} w(k) \\
d(k) & =C_{d} x_{d}(k)+D_{w} w(k),
\end{aligned}
$$

and the measurement noise $v(k)$ is white. Let

$$
\mathbf{E}\left[\begin{array}{l}
w(k) \\
v(k)
\end{array}\right]=0, \quad \mathbf{E}\left[\begin{array}{l}
w(k) \\
v(k)
\end{array}\right]\left[\begin{array}{l}
w(l) \\
v(l)
\end{array}\right]^{T}=R \delta_{k l},
$$

be the expected value and covariance of the white noise. When there is no outage, the entire system evolves as

$$
\begin{aligned}
{\left[\begin{array}{l}
x_{p}(k+1) \\
x_{c}(k+1) \\
x_{d}(k+1)
\end{array}\right]=} & \underbrace{\left[\begin{array}{ccc}
A_{p} & B_{u} C_{c} & B_{d} C_{d} \\
B_{c} C_{p} & A_{c} & 0 \\
0 & 0 & A_{d}
\end{array}\right]}_{A_{c l}}\left[\begin{array}{l}
x_{p}(k) \\
x_{c}(k) \\
x_{d}(k)
\end{array}\right] \\
& +\underbrace{\left[\begin{array}{cc}
B_{d} D_{w} & 0 \\
0 & B_{c} \\
B_{w} & 0
\end{array}\right]}_{N}\left[\begin{array}{l}
w(k) \\
v(k)
\end{array}\right] \\
u_{c}(k)= & \underbrace{\left[\begin{array}{lll}
0 & C_{c} & 0
\end{array}\right]}_{C_{c l}}\left[\begin{array}{l}
x_{p}(k) \\
x_{c}(k) \\
x_{d}(k)
\end{array}\right] .
\end{aligned}
$$

The optimal estimator of the state in (7) using measurements $u_{c}(k)$ is the Kalman filter,

$\hat{x}(k+1 \mid k)=A_{c l} \hat{x}(k \mid k-1)+K_{c l}\left[u_{c}(k)-C_{c l} \hat{x}(k \mid k-1)\right]$.

where

$$
\begin{aligned}
K_{c l}= & \left(A_{c l} P C_{c l}^{T}\right)\left(C_{c l} P C_{c l}^{T}\right)^{-1} \\
P= & A_{c l} P A_{c l}^{T}+N R N^{T} \\
& -\left(A_{c l} P C_{c l}^{T}\right)\left(C_{c l} P C_{c l}^{T}\right)^{-1}\left(A_{c l} P C_{c l}^{T}\right)^{T},
\end{aligned}
$$

see for example [14]. The optimal one-step-ahead prediction of $u_{c}(k)$ is $\hat{u}(k \mid k-1)=C_{c l} \hat{x}(k \mid k-1)$. Note that the Kalman filter (8) has the structure of the POC (3), and that optimal 
predictions of $u_{c}(k)$ based on measurements up until $k^{\prime}$ are generated by

$$
\hat{x}\left(k+1 \mid k^{\prime}\right)=A_{c l} \hat{x}\left(k \mid k^{\prime}\right), \quad \hat{u}\left(k \mid k^{\prime}\right)=C_{c l} \hat{x}\left(k \mid k^{\prime}\right),
$$

where $x\left(k^{\prime}+1 \mid k^{\prime}\right)$ is given by (8). Also the optimal predictor (10) has the form of a POC in prediction mode (4). We can conclude that the stochastically optimal POC is given by the choices $A=A_{c l}, K=K_{c l}$, and $C=C_{c l}$ under the given assumptions.

Remark 3: Note that we here consider the problem over an infinite time horizon. This is a good assumption if the communication outages are infrequent. If the time horizon is finite, the optimal filter gain $K_{c l}$ should be time varying, see [14].

Next, we compute the prediction error of the optimal POC. This error serves as a lower bound on the prediction error of other POCs. We can note that the state dimension of the optimal POC is large. How to approximate it is discussed in Section IV.

\section{A. Prediction Error}

It is easy to characterize the statistics of the prediction error $\Delta u(k)$ of the optimal POC. The Kalman filter gives unbiased estimates and thus $\mathbf{E} \Delta u(k)=0$ for all $k>k^{\prime}$. To compute the variance $\mathbf{E}|\Delta u(k)|^{2}$, we need the covariance of the state estimation error. Assuming that the Kalman filter has been in operation a long time before the outage at $k^{\prime}$, the covariance of $\Delta x(k)$ is given by the solution to the Riccati equation in (9), $\mathbf{E} \Delta x(k) \Delta x(k)^{T}=P$, where $\Delta x(k)=x(k)-\hat{x}(k \mid k-1)$ and $x(k)$ is the state vector in (7). The variance of the one-step-ahead prediction error is $\mathbf{E}|\epsilon(k)|^{2}=C_{c l} P C_{c l}^{T}$. When an outage occurs, the covariance of the state estimation and prediction error evolve for $k>k^{\prime}$ as

$$
\begin{aligned}
P(k+1) & =A P(k) A^{T}+N R N^{T}, \quad P\left(k^{\prime}\right)=P, \\
\mathbf{E}|\Delta u(k)|^{2} & =C_{c l} P(k) C_{c l}^{T} .
\end{aligned}
$$

The optimal POC minimizes the variance of the noise $\Delta u(k)$ in the model (5). How much effect this noise has on the plant of course depends heavily on its dynamics. If $\mathcal{P}$ is an unstable plant, even a small error $\Delta u(k)$ can harm the process since it is controlled in open loop during outage.

\section{REDUCED-ORDER POCS}

Let us represent the state-space POC by a linear operator $\hat{u}=G \epsilon$ on $\ell_{2}$, realized by

$$
G\left\{\begin{array}{l}
\hat{x}(k+1 \mid k)=A \hat{x}(k \mid k-1)+K \epsilon(k), \\
\hat{u}(k \mid k-1)=C \hat{x}(k \mid k-1), \hat{x}(k \mid k-1) \in \mathbb{R}^{n},
\end{array}\right.
$$

where $\epsilon(k)=u_{c}(k)-C \hat{x}(k \mid k-1)$. As has been discussed in Section III, the dimension $n$ of the state vector $\hat{x}(k \mid k-1)$ can be very large. This is the case if $K$ is the Kalman gain of the system, for example. It is of interest to investigate how the order of the POC can be reduced, and to get an understanding of the performance and complexity trade-off. The reduced POC serves both as an approximation to be implemented in devices with limited computational power as well as a method to determine whether there is something to gain or not by using a more advanced strategy to handle outages or not. Let us denote a reduced-order POC by a linear operator $\hat{u}_{r}=G_{r} \epsilon_{r}$ on $\ell_{2}$, with order $r<n$, realized by

$$
G_{r}\left\{\begin{array}{l}
\hat{x}_{r}(k+1 \mid k)=A_{r} \hat{x}_{r}(k \mid k-1)+K_{r} \epsilon_{r}(k), \\
\hat{u}_{r}(k \mid k-1)=C_{r} \hat{x}_{r}(k \mid k-1), \hat{x}_{r}(k \mid k-1) \in \mathbb{R}^{r},
\end{array}\right.
$$

where $\epsilon_{r}(k)=u_{c}(k)-C_{r} \hat{x}_{r}(k \mid k-1)$. One method for generating $G_{r}$ from a given $G$ is suggested next.

\section{A. Hankel Approximation of $G$}

Assuming, without loss of generality, that the outage occurs at $k^{\prime}=0$, we would like the reduced-order POC to produce an outage prediction $\hat{u}_{r}(k \mid 0)$ that is close to $\hat{u}(k \mid 0)$, for $k>0$, for any input sequence $u_{c}(k), k \leq 0$. Using operator notation, one way to formalize this requirement is to make the criterion

$$
\left\|\Gamma_{G}(I+G)^{-1}-\Gamma_{G_{r}}\left(I+G_{r}\right)^{-1}\right\|
$$

small. Here $\|\cdot\|$ is the induced $\ell_{2}$-norm, and the stable operators $(I+G)^{-1}$ and $\left(I+G_{r}\right)^{-1}$ map the input $u_{c}$ into the corrections $\epsilon$ and $\epsilon_{r}$, respectively. The Hankel operator $\Gamma_{G}$ is the past-input to future-output restriction of $G$, see for example [15]. That is, $\Gamma_{G}:=P_{+} G P_{-}$, where $P_{+}, P_{-}$are time-projection operators:

$$
\begin{aligned}
P_{+} u & =P_{+}(\ldots, u(2), u(1), u(0), u(-1), u(-2), \ldots) \\
& =(\ldots, u(2), u(1), 0,0,0, \ldots)) \\
P_{-} u & =P_{-}(\ldots, u(2), u(1), u(0), u(-1), u(-2), \ldots) \\
& =(\ldots, 0,0, u(0), u(-1), u(-2), \ldots),
\end{aligned}
$$

so that $\ell_{2}=P_{+} \ell_{2} \oplus P_{-} \ell_{2}$. The input-output map of $\Gamma_{G}$ is given by

$$
\hat{u}(k \mid 0)=\sum_{i=-\infty}^{0} C A^{k-i-1} K \epsilon(i)=\left(\Gamma_{G} \epsilon\right)(k), \quad k>0 .
$$

The reason for using approximation in the Hankel norm is that it measures how past inputs affects future outputs. This is natural since the POC is driven by the innovation signal $\epsilon(k)$ up until time $k^{\prime}=0$ after which it is no longer computable and we instead use $\epsilon(k)=0, k>k^{\prime}=0$. Since the POC output is only of interest after the outage we are effectively interested in how the past input signal $\epsilon(k)$ affects future estimates $\hat{u}(k)$. This exact property is captured by the Hankel norm.

Model order reduction is a well-studied topic, and there are many methods available, see for example the book [16]. The problem of making (13) small does not fit clearly to any of these methods, however. To be able to solve the problem, we note that the following bound holds:

$$
\begin{aligned}
\| \Gamma_{G}(I+G)^{-1} & -\Gamma_{G_{r}}\left(I+G_{r}\right)^{-1} \| \\
\leq & \left\|\left(\Gamma_{G}-\Gamma_{G_{r}}\right)\left(I+G_{r}\right)^{-1}\right\| \\
& +\left\|\Gamma_{G}\left((I+G)^{-1}-\left(I+G_{r}\right)^{-1}\right)\right\| .
\end{aligned}
$$

We will proceed by minimizing the first term of this upper bound. It turns out that it is then possible to use that solution to bound the second term, and thus to bound the error criterion (13).

Note that the rank of the Hankel operator is equal to the McMillan degree of the corresponding system, i.e., 
$\operatorname{rank} \Gamma_{G}=n$, if $(A, K, C)$ is a minimal realization. To make the first term in the upper bound (14) small, we therefore want to solve the problem

$$
\min _{\operatorname{rank} \Gamma_{G_{r}} \leq r} \sup _{\left\|\epsilon_{r}\right\|_{2} \leq 1}\left\|\left(\Gamma_{G}-\Gamma_{G_{r}}\right) \epsilon_{r}\right\|_{2}=: \gamma_{1}(r),
$$

where $\epsilon_{r}=\left(I+G_{r}\right)^{-1} u_{c}$. The approximation problem (15) can be solved using the famous AAK-lemma, see [17]. In particular, it is well-known that $\gamma_{1}(r)=\sigma_{r+1}(G)$ where $\sigma_{i}(G), i=1, \ldots, n$, are the Hankel singular values of the linear operator $G$, and methods for computing a state-space realization $\left(A_{r}, K_{r}, C_{r}\right)$ of the optimal $G_{r}^{*}$ are available, see [18], [19]. The Hankel singular values can be used to determine a suitable approximation order $r$.

Assume now we choose an optimal Hankel approximation $G_{r}^{*}$ of $G$ as the reduced POC. What can we then say about the size of the second term in the bound (14)? We have that $(I+G)^{-1}-\left(I+G_{r}\right)^{-1}=(I+G)^{-1}\left(G_{r}-G\right)\left(I+G_{r}\right)^{-1}$, and as has been shown in [18], [19], there is an optimal Hankel approximation $G_{r}^{*}$ such that

$$
\left\|G_{r}^{*}-G\right\| \leq \sum_{i=r+1}^{n} \sigma_{i}(G) .
$$

An upper estimate of the second term is therefore

$$
\begin{aligned}
& \left\|\Gamma_{G}\left((I+G)^{-1}-\left(I+G_{r}^{*}\right)^{-1}\right) u_{c}\right\|_{2} \\
& =\left\|\Gamma_{G}(I+G)^{-1}\left(G_{r}-G\right) \epsilon_{r}\right\|_{2} \leq \gamma_{2}(r)\left\|\epsilon_{r}\right\|_{2}
\end{aligned}
$$

where $\gamma_{2}(r)=\sigma_{1}(G)\left\|(I+G)^{-1}\right\| \sum_{i=r+1}^{n} \sigma_{i}(G)$, where we have used that the induced norm of $\Gamma_{G}$ is equal to $\sigma_{1}(G)$. This bound is expected to be quite conservative, since we have used the submultiplicative property of the induced norm and the upper bound (16) which is derived using the triangle inequality. The reason this bound is used is that the authors are not aware of any method to compute the induced norm $\left\|\Gamma_{G}(I+G)^{-1}\left(G_{r}-G\right)\right\|$. We summarize the above results in the following proposition.

Proposition 1: Suppose the system (12) is chosen as an optimal Hankel approximation $G_{r}^{*}$ of the stable system $G$ in (11). Then it holds for any input $u_{c} \in \ell_{2}$ that

$$
\left\|P_{+}\left(\hat{u}-\hat{u}_{r}\right)\right\|_{2}=\left\|\Gamma_{G} \epsilon-\Gamma_{G_{r}^{*}} \epsilon_{r}\right\|_{2} \leq \gamma(r)\left\|\epsilon_{r}\right\|_{2},
$$

where $\gamma(r)=\gamma_{1}(r)+\gamma_{2}(r)$ giving

$$
\gamma(r)=\sigma_{r+1}(G)+\sigma_{1}(G)\left\|(I+G)^{-1}\right\| \sum_{i=r+1}^{n} \sigma_{i}(G) .
$$

Proposition 1 shows that if $\sigma_{i}(G), i=r+1, \ldots, n$ are small, then $G_{r}^{*}$ is guaranteed to work well as a reducedorder POC. The bound can be used as follows: A user of the reduced-order POC can compute $\left\|\epsilon_{r}\right\|_{2}$, since this is the energy of the one-step ahead prediction error which is fed into $G_{r}^{*}$. If $\left\|\epsilon_{r}\right\|_{2}$ is small, it means that the environment is not very noisy, and the prediction works well. If then an outage occurs, we can be certain that the outage predictions $\hat{u}_{r}$ do not deviate from the full-order prediction $\hat{u}$ more than $\gamma(r)\left\|\epsilon_{r}\right\|_{2}$, under the same circumstances.

Remark 4: One restriction in Proposition 1 is that $G$ must be stable. This is not the case if an optimal POC has unstable modes in the disturbance model (6), for example. This can be handled by making a stable/anti-stable decomposition of
$G$, i.e., $G=G_{s}+G_{u}$, and then by approximating the stable part $G_{s}$ as above. The unstable term $G_{u}$ can then be added to the approximation $G_{s, r}^{*}$.

Remark 5: When $G$ is an optimal POC as described in Section III, it intuitively makes sense to make the reduction criteria $\left\|\Gamma_{G}-\Gamma_{G_{r}}\right\|$ small. The reason is that the correction $\epsilon=(I+G)^{-1} u_{c}$ that is applied to $\Gamma_{G}$ then is an innovation sequence, and is stochastically white noise. It therefore contains an equal amount of all frequencies and to make an unweighed criterion like $\left\|\Gamma_{G}-\Gamma_{G_{r}}\right\|$ small is natural.

\section{TANK EXAMPLE}

To exemplify the proposed method, we consider a tank process consisting of five identical tanks connected in series. This system is motivated from a floatation process in an ore concentrator at Boliden in Sweden, which is being investigated for wireless control within the SOCRADES project [1]. The control objective is to keep the level $h_{5}$ in the lowest tank around an equilibrium point despite load disturbances $d$ entering the system. The manipulated variable is the flow $u$ from the pump.

The individual tanks are modelled using mass balance and Bernoulli's law. Assuming that the tanks have cross sectional area $A=1 \mathrm{~m}^{2}$, outlet hole area $a=0.2 \mathrm{~m}^{2}$ and that the gravitational acceleration is $g=10 \mathrm{~m} / \mathrm{s}^{2}$, a linearized process model around the equilibrium $h_{i}^{0}=5 \mathrm{~m}$ and $u^{0}=2 \mathrm{~m}^{3} / \mathrm{s}$ is given by

$$
\left[\begin{array}{l}
\dot{h}_{1} \\
\dot{h}_{2} \\
\dot{h}_{3} \\
\dot{h}_{4} \\
\dot{h}_{5}
\end{array}\right]=\frac{1}{\tau}\left[\begin{array}{ccccc}
-1 & 0 & 0 & 0 & 0 \\
1 & -1 & 0 & 0 & 0 \\
0 & 1 & -1 & 0 & 0 \\
0 & 0 & 1 & -1 & 0 \\
0 & 0 & 0 & 1 & -1
\end{array}\right]\left[\begin{array}{l}
h_{1} \\
h_{2} \\
h_{3} \\
h_{4} \\
h_{5}
\end{array}\right]+\left[\begin{array}{l}
1 \\
0 \\
0 \\
0 \\
0
\end{array}\right](u+d)
$$

where $\tau=\frac{A}{a} \sqrt{\frac{2 h_{i}^{0}}{g}}=5 \mathrm{~s}$. The rise time of the water tank system is about $30 \mathrm{~s}$.

The controller $C(s)=\frac{s+\omega_{I}}{s}\left(\frac{\tau_{d} s+1}{\beta \tau_{d} s+1}\right)^{5} \frac{10 \omega_{I}}{s+10 \omega_{I}}$ is derived using loop-shaping, where $\omega_{I}=0.2, \beta=0.30$ and $\tau_{d}=$ 8.54. It gives cross-over frequency $\omega_{c}=0.22 \mathrm{rad} / \mathrm{s}$ and phase-margin $\varphi_{m}=60^{\circ}$.

The process and controller are sampled with period $T_{s}=$ $1 \mathrm{~s}$ and a disturbance model is derived to account for the disturbance $d$ as

$$
\begin{aligned}
x_{d}(k+1) & =x_{d}(k)+w(k) \\
d(k) & =x_{d}(k) \\
\mathbf{E} w(k)^{2} & =0.01
\end{aligned}
$$

Combining the models for the process, controller and disturbance as in (7) one get a closed-loop system with McMillan degree $n=n_{p}+n_{c}+n_{d}=5+7+1=13$.

\section{A. Optimal POC}

The optimal POC $G$ for the closed-loop tank system is given by (8) with degree $n=13$. We choose $R=$ $\operatorname{diag}\left(\left[10^{-2}, 10^{-4}\right]\right)$, and simulate the system under the following scenario:

$k=0$ : The system starts at rest and the disturbance $d$, generated according to (19), starts to act on the system. $k=40:$ A communication outage occur between controller and actuator and the POC is activated. 


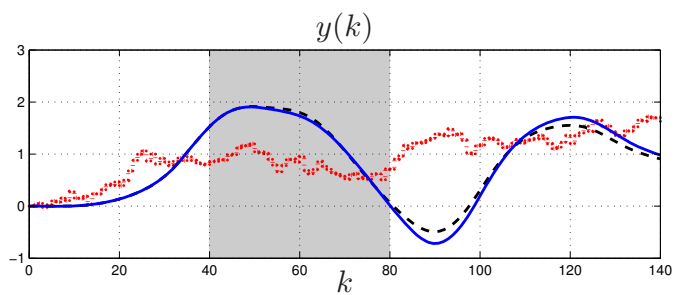

$u(k)$

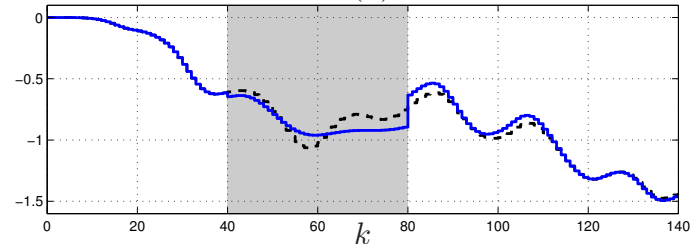

(a) optimal POC

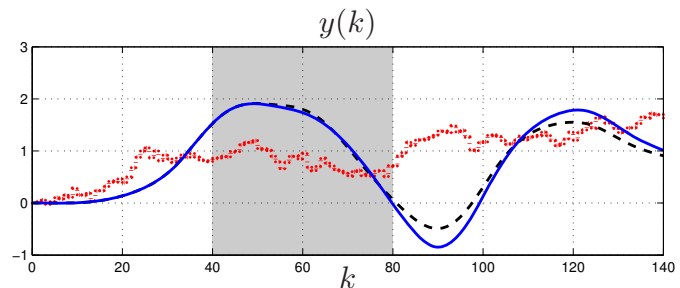

$u(k)$

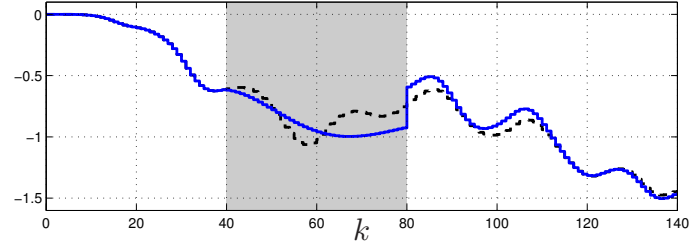

(c) reduced POC with $r=3$

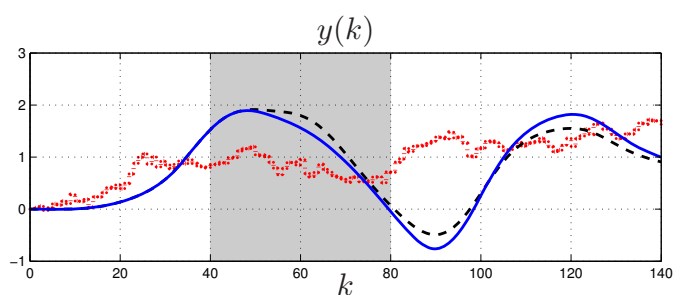

$u(k)$

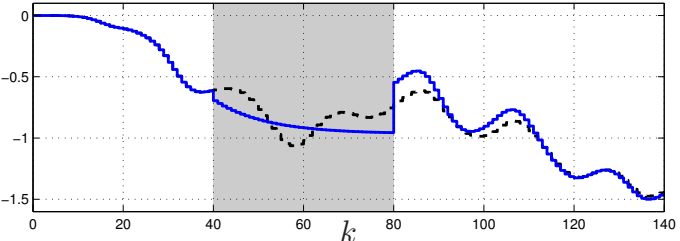

(b) reduced POC with $r=1$

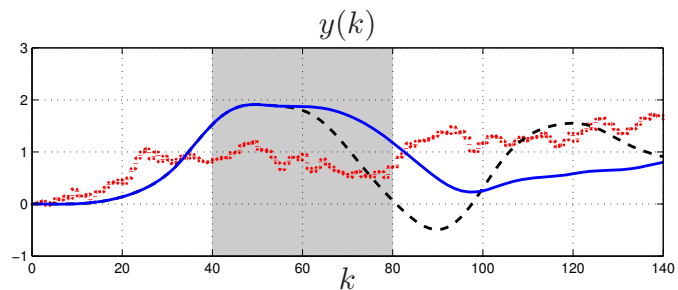

$u(k)$

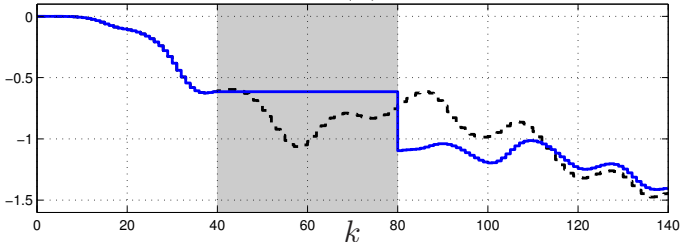

(d) hold POC

Fig. 3. Comparison of POC behavior (solid) with Nominal behavior (dashed) under disturbance $d$ (dotted)

$k=80:$ Communication is restored and the controller starts to actuate the system back into rest.

The resulting simulated behavior of $G$ is shown in Fig. 3(a). At time $k=0$ the optimal POC is initialized to have the same state as the true system and the prediction is perfect. However at the same instant the disturbance starts acting and the system states start to diverge, as a result so does the estimation error. Effectively what now happens is that the Kalman filter in the POC starts to estimate the variance of the random-walk disturbance $d$ via the internal model. At $k=40$ communication between the controller and actuator is lost, hence so is the feedback into the predictor, and the optimal POC starts to evolve in open loop predicting control signals. If the state estimate in the optimal POC has converged the prediction will be perfect, as long as the disturbance does not change under the outage. However, if the estimate has not fully converged, as is the case in this example, the prediction will start to diverge. Still, one can observe that the prediction error is small for the first 15 samples resulting in a very small deviation in the output compared to the nominal case.

\section{B. Hankel approximation}

As discussed previously in the paper, it is often desirable to have a POC of low order and therefore a reduced-order approximation of the optimal POC $G$ is desired. First it is observed that the disturbance model (19) contains an integration and that $G$ hence is not asymptotically stable. To handle this a stable/anti-stable decomposition of $G$ is made as $G=G_{s}+G_{u}$ and reduction is made on the stable part $G_{s}$ only, see Remark 4. To determine a proper reduction order $r$ the singular values of $G_{s}$, shown in Fig. 4, are studied. As seen there are significant drops between $\sigma_{1}\left(G_{s}\right)$ and $\sigma_{2}\left(G_{s}\right)$, between $\sigma_{3}\left(G_{s}\right)$ and $\sigma_{4}\left(G_{s}\right)$ and between $\sigma_{5}\left(G_{s}\right)$ and $\sigma_{6}\left(G_{s}\right)$, indicating that a good choice of the reduction order $r$ is to choose $r$ in the set $\{1,3,5\}$. Performing optimal Hankel norm approximation on $G_{s}$ of order $r$ one gets $G_{s, r}^{*}$ and the reduced POC as $G_{r}^{*}=G_{s, r}^{*}+G_{u}$ of order $r+1$ since $G_{u}$ only contains the integrator state from the disturbance model.

A quantitative bound $\gamma(r)$ on the reduction is given in Proposition 1. For the given example the bounds for the suggested choices of $r$ are presented in the table below together with the true norm of $\left\|P_{+}\left(\hat{u}-\hat{u}_{r}\right)\right\|_{2}$ and the contribution from the partial bound $\gamma_{1}(r)$ in (15).

\begin{tabular}{c|ccc|c}
$r$ & $\left\|\epsilon_{r}\right\|_{2}$ & $\gamma_{1}(r)\left\|\epsilon_{r}\right\|_{2}$ & $\gamma(r)\left\|\epsilon_{r}\right\|_{2}$ & $\left\|P_{+}\left(\hat{u}-\hat{u}_{r}\right)\right\|_{2}$ \\
\hline 1 & 0.35 & 0.77 & 11.87 & 0.39 \\
3 & 0.29 & 0.25 & 2.99 & 0.26 \\
5 & 0.32 & 0.03 & 0.25 & 0.02
\end{tabular}




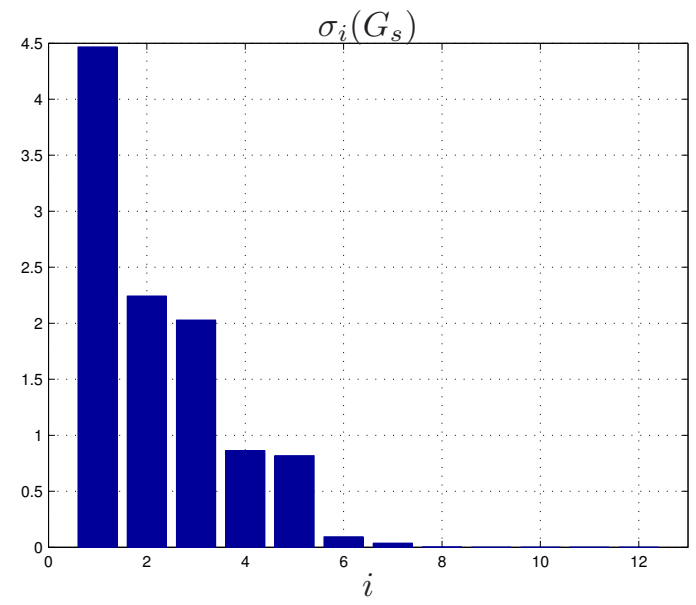

Fig. 4. Hankel singular values of the stable modes in the optimal POC

As seen, in this example, the bound imposed by $\gamma_{1}(r)$ is close to the true value of $\left\|P_{+}\left(\hat{u}-\hat{u}_{r}\right)\right\|_{2}$ whereas the upper bound given by $\gamma(r)$ is significantly larger. This is due to the conservative derivations of the component $\gamma_{2}(r)$ from (17). Evaluating the reduced POCs with reduction order $r=1$ and $r=3$ on the same simulation scenario as before one gets the results in Fig. 3(b) resp. Fig. 3(c).

At $k=0$ the system starts at rest in the origin and the reduced POCs are initialized accordingly to $x_{r}(0)=0$. As before the disturbance $d$ starts to act on the system immediately causing it to diverge and the POCs start estimating the states. The state associated with the disturbance model will now, apart from the disturbance, accommodate the errors due to the model reduction. When communication is lost at $k=40$ the POCs will as before evolve in open loop predicting the control signal.

Studying the reduced POC of reduction order $r=1$ in Fig. 3(b) one can see that the prediction error as expected is larger than for the optimal POC, this is due both to the model approximation error and the fact that the estimator has not fully converged when the outage occur. If one instead studies the reduced POC of reduction order $r=3$ in Fig. 3(c) the prediction error is smaller than for order $r=1$, although the difference is not significant. However, if one studies the output it is clear that the output tracking performance for the reduced POC with $r=3$ is almost identical to the optimal POC.

\section{Hold approximation}

A common way to compensate for outages is to use a hold POC. That is to use the last known value of the control signal $u_{c}(k)$ as the one-step-ahead prediction. This POC is a firstorder approximation of the optimal POC with $A_{r}=K_{r}=$ $C_{r}=1$ in (12). The resulting simulation over the studied scenario from using this predictor is shown in Fig. 3(d). As seen the control signal tracking is poor, resulting in a large drift in the output compared to the nominal closedloop behavior.

\section{Conclusions And Future Work}

In this paper, we have proposed predictive outage compensation as a method to reduce the influence of communication outages in networked control systems. In particular, we derived an optimal prediction scheme, and also showed how the order of the predictor can be reduced by means of Hankel-norm approximation. A priori approximation error bounds of the predictor were derived, and the method was also demonstrated on a simulated tank system.

The optimal predictor estimates the state of the entire closed-loop system. An interesting topic for future work is to find alternative methods of using this estimate. One could use a separate open-loop controller in outage, for instance. Another problem of both practical and theoretical importance is how to generate controls to minimize the bump in the control signal after a communication outage.

\section{REFERENCES}

[1] SOCRADES, Integrated Project. EU 6th Framework Programme, http://www.socrades.eu/.

[2] A. Willig, M. Kubisch, C. Hoene, and A. Wolisz, "Measurements of a wireless link in an industrial environment using an IEEE 802.11compliant physical layer," IEEE Transactions on Industrial Electronics, vol. 49, no. 6, pp. 1265-1282, 2002.

[3] X. Liu and A. Goldsmith, "Wireless network design for distributed control," in Proceedings of the IEEE Conference on Decision and Control, Atlantis, Paradise Island, Bahamas, 2004, pp. 2823-2829.

[4] Q. Ling and M. Lemmon, "Optimal dropout compensation in networked control systems," in Proceedings IEEE Conference on Decision and Control, Maui, HI, USA, 2003.

[5] J. Hespanha, P. Naghshtabrizi, and Y. Xu, "A survey of recent results in networked control systems," Proceedings of the IEEE, special issue, vol. 95, no. 1, pp. 138-192, 2007.

[6] L. Schenato, B. Sinopoli, M. Franceschetti, K. Poolla, and S. Sastry, "Foundations of control and estimation over lossy networks," Proceedings of the IEEE, special issue, vol. 95, no. 1, pp. 163-187, 2007.

[7] L. Schenato, "To zero or to hold control inputs with lossy links?" IEEE Transactions on Automatic Control, vol. 54, no. 5, pp. 1093 1099, May 2009.

[8] S. Graham and P. R. Kumar, "The convergence of control, communication, and computation," in pp. 458-475, Proceedings of PWC 2003: Personal Wireless Communication, Lecture Notes in Computer Science, Volume 2775. Springer-Verlag, Heidelberg, 2003.

[9] K.-E. Årzén, A. Bicchi, G. Dini, S. Hailes, K. H. Johansson, J. Lygeros, and A. Tzes, "A component-based approach to the design of networked control systems," European Journal of Control, vol. 2-3, pp. 261-279, 2007, Invited Paper.

[10] V. Gupta and N. C. Martins, "On stability in the presence of analog erasure channels," in Proceedings IEEE Conference on Decision and Control, Cancun, Mexico, December 2008.

[11] A. Bemporad, "Predictive control of teleoperated constrained systems with unbounded communication delays," in Proceedings IEEE Conference on Decision and Control, Tampa, FL, December 1998.

[12] D. Quevedo, E. Silva, and G. Goodwin, "Control over unreliable networks affected by packet erasures and variable transmission delays," IEEE Journal on Selected Areas in Communication, vol. 26, no. 4, pp. 672-685, May 2008.

[13] E. Henriksson, H. Sandberg, and K. H. Johansson, "Predictive compensation for communication outages in networked control systems," in Proceedings IEEE Conference on Decision and Control, Cancun, Mexico, December 2008.

[14] B. D. O. Anderson and J. B. Moore, Optimal Filtering. Dover Publications, 2005.

[15] K. Zhou, J. Doyle, and K. Glover, Robust and Optimal Control. Upper Saddle River, New Jersey: Prentice Hall, 1996.

[16] G. Obinata and B. Anderson, Model Reduction for Control System Design. London, UK: Springer-Verlag, 2001

[17] V. Adamjan, D. Arov, and M. Krein, "Analytic properties of Schmidt pairs for a Hankel operator and the generalized Schur-Takagi problem," Math. USSR Sbornik, vol. 15, no. 1, pp. 31-73, 1971.

[18] K. Glover, "All optimal Hankel-norm approximations of linear multivariable systems and their $L_{\infty}$-error bounds," International Journal of Control, vol. 39, pp. 1115-1193, 1984.

[19] G. Gu, "All optimal Hankel-norm approximations and their error bounds in discrete-time," International Journal of Control, vol. 78 , no. 6, pp. 408-423, 2005. 